\title{
Interactive Databases for Cardiac Rehabilitation: the "CardioDB" Electronic Archive elaborated by the Don Carlo Gnocchi Foundation
}

\author{
AA Conti ${ }^{1,2}, \mathrm{R}^{2}$ Molino Lova ${ }^{1}$, A Conti $^{3}$, E Ferrari $^{1}$, \\ C Macchi ${ }^{1,2}$, GF Gensini ${ }^{1,2}$ \\ ${ }^{1}$ Don Carlo Gnocchi Foundation, Florence \\ ${ }^{2}$ Dept Critical Medicine and Surgery, Univ Florence, Florence \\ ${ }^{3}$ Dept Clinical Pathophysiology, Univ Florence, Florence, Italy
}

\begin{abstract}
The main aim of Cardiac Rehabilitation is the reduction of new clinical events through cardiovascular risk factors control. In the Florence Cardiologic Unit of the Don Gnocchi Foundation Institute approximately 350 patients are managed each year.

To perform a full follow-up analysis of the patients the data included in a traditional database are currently being transferred into the newly developed electronic archive of the Don Gnocchi Foundation. Through the insertion of the maximum possible quantity of numerical information as soon as it is available, clinical work is speeded up and a quality control of inserted data is possible.

Such a system guarantees the possibility of producing complete diagnostic records by introducing numerical data; it also provides reports accounting for the outcome of treatments and even the discharge letter.

The CardioDB is also functional for scientific research purposes since its quantitative content may be immediately accessed, processed and analyzed in statistical terms.
\end{abstract}

\section{Introduction}

According to the World Health Organization (1993) cardiac rehabilitation is "the sum of activities and interventions required to ensure the best possible physical, mental, and social conditions so that patients with chronic or post-acute cardiovascular disease may, by their own efforts, preserve or resume their proper place in society and lead an active life" [1].

\subsection{Aims of cardiac rehabilitation}

In this perspective, cardiac rehabilitation is aimed at:

a) short-term prevention of disability that may result from heart disease, by appropriate evidence-based prescription of drugs and physical exercise; b) long-term prevention of subsequent cardiovascular events, by strict control of modifiable cardiovascular risk factors [2-10].

A testing of the achievement of these goals ("product indicators"), as well as an outline of user characteristics of any single Rehabilitation Centre ("case mix"), are essential for National Health Service planning. Furthermore, the possibility of correlating clinical and instrumental data, treatment protocols and outcomes is mandatory for research in the cardiac rehabilitation field.

The above considerations strongly suggest the need for an effective computer tool.

\subsection{Traditional and computerized Databases}

Currently available databases for medical use may be classed into two categories:

a) computerized medical files;

b) traditional databases.

Computerized medical files effectively replace paper medical files and have the advantage of allowing quick consultation of previously recorded data and of printing paper documents, when needed. Nevertheless, data are filed as texts and are not suitable for further processing.

On the contrary, data recorded in traditional databases are filed as figures and can be easily processed. Furthermore, traditional databases have substantial limits referable to three issues:

a) their use implies an overload that is negatively perceived on the part of their users, since the latter have to fill in electronic formats after having already filled in paper documents;

b) their informative content is often incomplete and/or unreliable because of the lack of an effective quality control system for inserted data;

c) the majority of available data are stocked in text format, precluding future extraction of numerical information for statistical analysis. 


\section{The Florence Don Gnocchi Cardiologic Unit}

In the Florence Cardiologic Unit of the Don Carlo Gnocchi Foundation approximately 350 patients are managed each year, amounting to over 1,000 patients in a three-year time period.

In order to perform a full follow-up analysis of these patients the data included in a traditional database are currently being transferred into the newly developed electronic archive of the Don Carlo Gnocchi Foundation.

In planning and elaborating the "CardioDB" of the Don Carlo Gnocchi Foundation the previously mentioned problems have been considered so as to solve them by designing a system allowing the direct electronic insertion of data, with subsequent automatic print-out of paper documents.

Grids for all recordable data during the hospital stay have been foreseen and implemented. Inserted data include personal, diagnostic, therapeutic, and functional evaluation typologies.

Through the insertion of the maximum possible quantity of numerical information as soon as it is available, clinical work is speeded up and a quality control of inserted data is possible.

\section{The "CardioDB"}

In detail, a systematic attempt has been made to solve the intriguing challenge of bridging the gap between texts and figures by completely reversing the procedure: no longer a first filling up of paper documents (texts) and then an entering of data into the database (figures), but solely the entering of data into the database with subsequent temporary automatic transformation of figures into texts and printing of paper documents. This strategy results both in a saving of users' time, because there is no longer any need to fill up paper documents, and in a check of the completeness and correctness of entered data, because users read automatically generated and printed paper documents before signing them.

\subsection{Operative strategy}

This operative strategy required a great deal of time, in particular in order to decode numbers, included in the CardioDB, into character strings necessary to constitute texts acceptable from a linguistic point of view. However, the advantages obtained have proved to be remarkable. It is much faster to "click" on one of the electronic fields rather than to write the clinical history of the patient, or his/her discharge report (the fields of the CardioDB may almost all be ticked off and have default values such as
"0" or "no" or "blank"). Moreover, it allows the performance of a constant quality control of the inserted data.

Another relevant decision taken in the development process of the CardioDB was the selection of the platform to be adopted so as to elaborate the electronic archive. ACCESS, included in the package OFFICE 2000 of Microsoft, was selected, because of the wide diffusion of this program and its relative capacities in the mandatory periodical updating and upkeeping operations of the CardioDB. Moreover, an interactive electronic archive developed by using ACCESS may easily be exported onto the SQL SERVER, another Microsoft product, which appears to be one of the safest and most solid programs currently on the market.

This choice has however entailed the onset of another problem. ACCESS automatically records every modification, either accidental or deliberate, and therefore it is extremely easy to modify, in an involuntary manner, the contents of the database. In order to solve this problem in the CardioDB two series of forms have been created, one for the insertion of data, of a blue colour, and another for the visualization and the possible modification of already inserted data, of a green colour, that makes reference to a system of supporting variables, and that, before being permanently recorded into tables, requires a double consent ("Save" and "Are you sure you want to save data?") [11-13]. This safeguard too necessitated a lot of time, due to the fact that the real variables are approximately 1,000 , and besides them, there are 1,000 supporting variables for the insertion and 1,000 supporting variables for the visualization and the possible modification, summing up to a total of around 3,000 variables to be managed.

\subsection{Texts and reports}

The texts regarding the history of patients, the discharge report and a number of chemical, instrumental and clinical examinations performed in the course of the rehabilitative period (EKG, chest $\mathrm{XR}$, echocardiography and Holter EKG) have been generated by using the Mail Merge procedure of Word, thus obtaining potentially modifiable texts.

Reports regarding different examinations performed in the course of the rehabilitative period (chemical laboratory tests, treadmill tests, 6 minutes' walk corridor test), concerning the physiotherapic treatment, the case mix and the product indicators, have been generated directly from ACCESS, and they are not modifiable.

As far as regards other more complex statistical processes than those included in the case mix and in the product indicators, the data to process may be exportable, after performing a query directly from ACCESS and an EXCEL sheet, and this operation may be performed from 
any commercial statistics package (e.g.: Stata, SAS).

\section{The Operative Manual}

An operative manual has been developed for every instruction. It contains the overall structure of the CardioDB and the specific operative instructions regarding the individual forms.

\subsection{Overall structure}

Six administrative typologies, four clinical typologies and four patient typologies have been foreseen in the elaboration phase. In effect, the Florentine centre of the Don Carlo Gnocchi Foundation is a complex structure and its Cardiologic Unit foresees various modalities of patient admission and of hospital stay, including ordinary and outpatient clinics and day hospital, all targeted to cardiovascular rehabilitation. With specific reference to the typologies of patients admitted, the four abovementioned profiles are represented by:

1) patients who have undergone cardiac surgery,

2) patients who have undergone percutaneous coronary interventions,

3) patients with myocardial infarction,

4) patients with chronic heart failure.

\subsection{Individual forms}

The CardioDB is made up of 21 forms organized on the logic of use according to the phase of health treatment. Each form is constituted by two parts: anagraphic data and administrative data. The 21 forms are the following: anagraphic data; clinical typology of health treatment (subdivided into clinical profile and control of cardiovascular risk factors); overall history of the patient; overall remote cardiac anamnesis; overall proximal cardiac anamnesis and invasive examinations; interventional proximal cardiac anamnesis; cardiac surgery proximal anamnesis; admission treatment; case mix (subdivided into indications for cardiovascular rehabilitation, clinical features and eventual research study protocol regarding the patient); EKG menu; chest $\mathrm{XR}$ menu; chemical laboratory tests menu; rest echocardiography menu; stress echocardiography menu; Holter EKG menu; treadmill test menu; 6 minutes' walk corridor test menu; different questionnaires; physiotherapic report; in-hospital clinical course; discharge report. Specifically, questionnaires include items regarding the physical disability status, the mental state, depression and anxiety evaluation and the general quality of life of cardiovascular patients of the Florentine centre of the Don Carlo Gnocchi Foundation.

\section{Conclusions}

In conclusion, the vast majority of data are recorded in the CardioDB of the Don Carlo Gnocchi Foundation as "numbers" and temporarily transformed into "text" only in the phase of automatic print-out. To avoid incompleteness in the CardioDB each datum may be inserted only when the previous boxes of the grids have been filled in.

A similar system therefore guarantees the possibility of producing not only complete diagnostic records, merely by introducing numerical data, and filling in grids immediately after the performance of the relative examination, but it also provides reports accounting for the outcome of treatments and even the discharge letter that usually takes a considerable amount of time when traditional recording systems are used.

Moreover, the CardioDB is also functional for scientific research purposes since its quantitative content may be immediately accessed and consequently processed and analyzed in statistical terms.

\section{Acknowledgements}

The Authors would like to thank Professor Luisa Camaiora, B.A., M.Phil., for her correction of the English.

\section{References}

[1] World Health Organization. Rehabilitation after cardiovascular diseases, with special emphasis on developing countries. Report of a WHO Expert Committee. World Health Organization Technical Report Series 1993; 831: 1-122.

[2] Haskell WL, Alderman EL, Fair JM, Maron DJ, Mackey SF, Superko HR, Williams PT, Johnstone IM, Champagne MA, Krauss RM. Effects of intensive multiple risk factors reduction on coronary atherosclerosis and clinical cardiac events in men and women with coronary artery disease. The Stanford Coronary Risk Intervention Project (SCRIP). Circulation 1994; 89: 975-990.

[3] Balady GJ, Ades PA, Comoss P, Limacher M, Pina IL, Southard D, Williams MA, Bazzarre T. Core components of cardiac rehabilitation/secondary prevention programs: A statement for healthcare professionals from the American Heart Association and the American Association of Cardiovascular and Pulmonary Rehabilitation Writing Group. Circulation 2000; 102: 1069-1073.

[4] Ades PA. Cardiac rehabilitation and secondary prevention of coronary heart disease. The New England Journal of Medicine 2001; 345: 892-902.

[5] Thompson PD, Buchner D, Pina IL, Balady GJ, Williams MA, Marcus BH, Berra K, Blair SN, Costa F, Franklin B, Fletcher GF, Gordon NF, Pate RR, Rodriguez BL, Yancey AK, Wenger NK. Exercise and physical activity in the prevention and treatment of atherosclerotic cardiovascular disease: a statement from the Council on Clinical Cardiology (Subcommittee on Exercise, Rehabilitation, 
and Prevention) and the Council on Nutrition, Physical Activity, and Metabolism (Subcommittee on Physical Activity). Circulation 2003; 107: 3109-3116.

[6] Thompson RL, Summerbell CD, Hooper L, Higgins JP, Little PS, Talbot D, Ebrahim S. Dietary advice given by a dietitian versus other health professionals or self-help resources to reduce blood cholesterol. Cochrane Database Systematic Review 2003; Issue 3, CD 001366.

[7] Scottish Intercollegiate Guidelines Network. \#57 Cardiac Rehabilitation. A National Clinical Guideline. 2002. Available online at http://www.sign.ac.uk/pdf/sign57.pdf (accessed on August 26th, 2005).

[8] Giannuzzi P, Saner H, Bjornstad H, Fioretti P, Mendes M, Cohen-Solal A, Dugmore L, Hambrecht R, Hellemans I, McGee H, Perk J, Vanhees L, Veress G. Secondary prevention through cardiac rehabilitation: position paper of the Working Group on Cardiac Rehabilitation and Exercise Physiology of the European Society of Cardiology. Eur Heart J 2003; 24: 1273-1278.

[9] American Association of Cardiovascular \& Pulmonary Rehabilitation - AACVPR. Guidelines for Cardiac
Rehabilitation and Secondary Prevention Programs, 2004, 4th edition, Human Kinetics Publishers: Champaign, Illinois (USA).

[10] Gensini GF, Conti AA. Rehabilitation in the elderly: differentiated strategies and objectives? Ital Heart J 2003; 4 Suppl 8: 73S-75S.

[11] Dobson R. Programmare Microsoft ACCESS 2002. Mondadori Informatica, Milano, 2001.

[12] Perry G. Visual Basic 6. Apogeo, Milano, 1998.

[13] Cassel P, Eddy C, Price J. Access 2002. Apogeo, Milano, 2002.

Address for correspondence

Andrea A. Conti,

Department of Critical Medicine and Surgery, University of Florence, Viale Morgagni 85, I-50134 Firenze, Italy.

Tel.: +39-055-417928;

Fax: +39-055-4379384;

E-mail: aa.conti@dac.unifi.it 